\title{
OPTIMAL DESIGN OF FLEXIBLE SHALLOW SHELLS ON ELASTIC FOUNDATION
}

Leonid Yulianovich Stupishin

Southwest State University, Kursk, Russia

Alexandr Georgiyevich Kolesnikov

Southwest State University, Kursk, Russia

Konstantin Evgenyevich Nikitin

Southwest State University, Kursk, Russia

This paper contains analysis of optimal forms of a geometric nonlinear (flexible) shallow shells based on an elastic foundation. Rise of arch in the center of the shell, width, length and type of support are given. The design variable is taken to be the thickness of the shallow shell, the form of the middle surface forming and the characteristic of elastic foundations. Critical force coefficient and stress of shells are calculated by Bubnov-Galerkin.

Key words: Shallow shells, Nonlinearity, Elastic foundation, Critical force, Strength, Variable form

\section{INTRODUCTION}

Shallow shell on elastic foundation is promising structures. They can be used as the foundations of buildings and structures, spans of bridges, highways constructions. Often designers solve problems not only to calculate such a structure, but also to find the best of its optimal parameters. Therefore, the development of methods of their analysis is an urgent task. Currently, most of these structures are designed with a software based on the finite element method. It is convenient model for engineering design gives good results only for linear problems. In the case of non-linear problems, you can get results, the accuracy of which is difficult to assess.

The result is highly dependent on the type and numbers of finite element. Increasing the number of finite elements does not lead to accuracy. In addressing some of the challenges of designing, analyzing and optimizing designs, more results that are accurate are obtained by using numerical methods. Therefore, the development of numerical methods for the design of structures operating in the nonlinear stage is necessary, including to be compared with the results of finiteelement method.

\section{EQUATIONS FOR THE DETERMINATION OF CRITICAL FORCE COEFFICIENT AND STRESS FOR SHALLOW SHELLS ON AN ELASTIC FOUNDATION}

Equations for the determination of critical force coefficient and stress for shallow shells on an elastic foundation given in [01, 02]:

$$
\left\{\begin{array}{l}
\frac{1}{E h} \nabla^{2} \nabla^{2} \varphi+k_{y} \frac{\partial^{2} w}{\partial x^{2}}+k_{x} \frac{\partial^{2} w}{\partial y^{2}}-2 k_{x y} \frac{\partial^{2} w}{\partial x \partial y}+\frac{\partial^{2} w}{\partial x^{2}} \frac{\partial^{2} w}{\partial y^{2}}-\left(\frac{\partial^{2} w}{\partial x \partial y}\right)^{2}=0 \\
D \nabla^{2} \nabla^{2} w-\frac{\partial^{2} \varphi}{\partial y^{2}}\left(k_{x}+\frac{\partial^{2} w}{\partial x^{2}}\right)-\frac{\partial^{2} \varphi}{\partial x^{2}}\left(k_{y}+\frac{\partial^{2} w}{\partial y^{2}}\right)+ \\
+2 \frac{\partial^{2} \varphi}{\partial x \partial y}\left(k_{x y}+\frac{\partial^{2} w}{\partial x \partial y}\right)=Z+2 t \nabla^{2} w-k w
\end{array}\right.
$$

Where

$\varphi$ - effort function, $w$ - deflection function.

$k_{x} \approx \frac{\partial^{2} F}{\partial x^{2}}$

$k_{y} \approx \frac{\partial^{2} F}{\partial y^{2}}$

$F=F(x, y)$ - the equation of the middle surface of the shell,

$\mathrm{Z}$ - function loading,

$\mathrm{k}$ - modulus of elastic foundation reaction,

$\mathrm{t}$ - soil shear parameter,

$$
\begin{aligned}
& k=\frac{E_{0}}{1-V_{0}} \int_{0}^{H} \Psi^{\prime 2} d z, \\
& t=\frac{E_{0}}{4\left(1-V_{0}\right)} \int_{0}^{H} \Psi^{2} d z .
\end{aligned}
$$


Where:

$$
\begin{aligned}
& E_{0}=\frac{E_{s}}{1-v_{s}}, \\
& v_{0}=\frac{v_{s}}{1-v_{s}},
\end{aligned}
$$

$\mathrm{E}_{\mathrm{s}}$ and $\mathrm{V}_{\mathrm{s}}$ - elastic module and Poisson's ratio of the soil, $\mathrm{H}$ - depth of the soil stratum,

$\Psi(\mathrm{z})$ - function of cross distribution displacements.

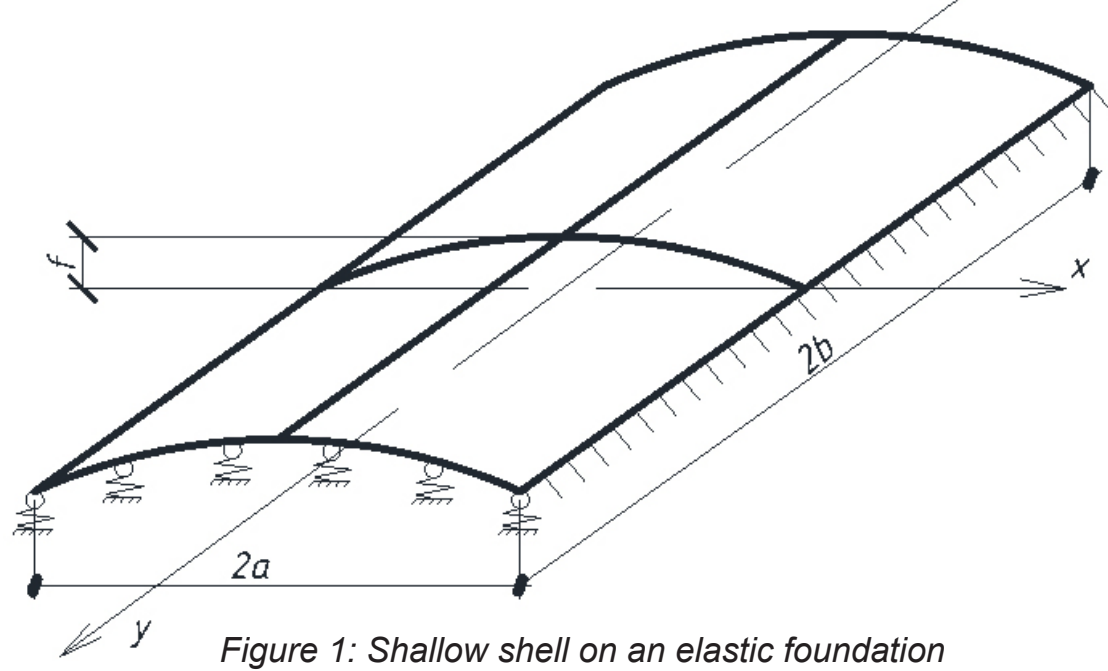

Function of cross distribution displacements:

$\Psi(z)=\frac{\operatorname{shy} \frac{H-z}{a}}{\operatorname{shy} \frac{H}{a}}$

Where:

$\mathrm{Y}$ - parameter that characterizes the vertical deformation profile within the soil continuum.

Equivalent stresses in the shell are found by the fourth stress hypothesis. Stress coefficient can be described by the equation [03, 04]:

$$
\sigma=\sqrt{\frac{1}{2}\left[\left(\overline{\sigma_{1}}-\overline{\sigma_{2}}\right)^{2}+\left(\overline{\sigma_{3}}-\overline{\sigma_{2}}\right)^{2}+\left(\overline{\sigma_{2}}-\overline{\sigma_{1}}\right)^{2}\right]}
$$

Where:

$\overline{\sigma_{1}}=\left|\frac{6}{t} \bar{D} \bar{B}\left(\frac{\partial^{2}}{\partial x^{2}} Z_{x} Z_{y}+v \frac{\partial^{2}}{\partial y^{2}} Z_{y} Z_{x}\right)\right|+\left|\bar{A} \frac{\partial^{2}}{\partial y^{2}} Z_{y} Z_{x} t\right|+$

$+\left|\frac{g}{t^{2}} \bar{D} \bar{B}\left(\frac{\partial^{3}}{\partial x^{3}} Z_{x} Z_{y}+\frac{\partial}{\partial x} Z_{x} \frac{\partial^{2}}{\partial y^{2}} Z_{y}\right)\right|$,

$\overline{\sigma_{2}}=\left|\frac{6}{t} \bar{D} \bar{B}\left(v \frac{\partial^{2}}{\partial x^{2}} Z_{x} Z_{y}+\frac{\partial^{2}}{\partial y^{2}} Z_{y} Z_{x}\right)\right|+\left|\bar{A} \frac{\partial^{2}}{\partial x^{2}} Z_{x} Z_{y} t\right|+$

$+\left|\frac{g}{t^{2}} \bar{D} \bar{B}\left(\frac{\partial^{3}}{\partial y^{3}} Z_{y} Z_{x}+\frac{\partial}{\partial y} Z_{y} \frac{\partial^{2}}{\partial x^{2}} Z_{x}\right)\right|$.

$\overline{\sigma_{3}}=\left|\frac{6}{t}(1-v) \bar{D} \bar{B} \frac{\partial}{\partial x} Z_{x} \frac{\partial}{\partial y} Z_{y}\right|+\left|\bar{A} \frac{\partial}{\partial x} Z_{x} \frac{\partial}{\partial H} Z_{y} t\right|$.

$$
\begin{aligned}
& \bar{D}=\frac{D}{E h^{3}}, \\
& D=\frac{E h^{3}}{12\left(1-v^{2}\right)}, \\
& \bar{A}=\frac{A \cdot a^{2}}{E f_{0}^{5}}, \\
& \bar{B}=\frac{B \cdot a^{2}}{f_{0}^{3}}, \\
& g=\frac{f_{0}}{a},
\end{aligned}
$$

$A=-\frac{1}{J_{1}}\left(B J_{2}+B^{2} J_{3}\right)$,

$B=\frac{1}{6 C_{1}}\left(36 C_{3} C_{2} C_{1}+108 q C_{1}^{2}-8 C_{2}^{3}+12 \sqrt{3}\left(4 C_{3}^{3} C_{1}-C_{3}^{2} C_{2}^{2}+18 C_{3} C_{2} C_{1} q+\right.\right.$ $\left.\left.+27 q^{2} C_{1}^{2}-4 q C_{2}^{3}\right)^{1 / 2} C_{1}\right)^{1 / 3}-\frac{2}{3}\left(3 C_{3} C_{1}-C_{2}^{2}\right) /\left(C_{1}\left(36 C_{3} C_{2} C_{1}+108 q C_{1}^{2}-\right.\right.$ $-8 C_{2}^{3}++12 \sqrt{3}\left(4 C_{3}^{3} C_{1}-C_{3}^{2} C_{2}^{2}+18 C_{3} C_{2} C_{1} q+\right.$

$$
\left.\left.\left.+27 q^{2} C_{1}^{2}-4 q C_{2}^{3}\right)^{1 / 2} C_{1}\right)^{1 / 3}-\frac{1}{3} \frac{C_{2}}{C_{3}}\right) \text {. }
$$

$C_{1}=2 E h \frac{J_{3}^{2}}{J_{1} J_{4}}$,

$C_{2}=3 E \frac{J_{3} J_{2}}{J_{1} J_{4}}$

$C_{3}=D \frac{J_{1}^{2}}{J_{1} J_{4}}+E h \frac{J_{2}^{2}}{J_{1} J_{5}}$. 


$$
\begin{aligned}
& J_{1}=\int_{-s}^{a} \int_{-b}^{b}\left(\nabla^{2} \nabla^{2} \bar{w}\right) \bar{w} d x d y \\
& J_{2}=\int_{-s}^{x} \int_{-b}^{b}\left(\frac{\partial^{2} F}{\partial y^{2}} \frac{\partial^{2} \bar{W}}{\partial x^{2}}+\frac{\partial^{2} F}{\partial x^{2}} \frac{\partial^{2} \bar{W}}{\partial y^{2}}-2 \frac{\partial^{2} F}{\partial x \partial y} \frac{\partial^{2} \bar{W}}{\partial x \partial y}\right) \bar{w} d x d, \\
& J_{3}=\int_{-s}^{s} \int_{-\infty}^{b}(\Delta \bar{W} \bar{W} d x d y \\
& J_{4}=\int_{-s}^{s} \int_{-b}^{b} Z \bar{w} d x d y \\
& J_{5}=\int_{-s-b}^{s} \int_{-b}^{b}\left(2 \bar{N}^{2} \bar{W}-k \bar{w}\right) \bar{w} d x d y
\end{aligned}
$$

$\mathrm{h}$ - shell thickness,

E - elastic module,

$v$ - Poisson's ratio,

A, B - unknown of Bubnov-Galerkin method,

Zx, Zy - beam function.

Critical force coefficient for shallow shells on an elastic foundation can be described by the equation:

$$
p_{c r}=\frac{2}{27} \frac{1}{C_{1}^{2}}\left[\left(C_{1}^{2}-3 C_{1} C_{3}\right)^{3 / 2}+C_{2}\left(C_{2}^{2}-\frac{9}{2} C_{1} C_{3}\right)\right] \text {. }
$$

The results obtained by this method were compared with other techniques. Table 1 shows the value for bending moment of a $9150 \times 12200 \times 150$ $\mathrm{mm}$ spherical metal shell. The rise of arch in the centre of the shell $=300 \mathrm{~mm}$, elastic module of the soil Es $=6895 \mathrm{~Pa}$ for all curve. Depth of the soil stratum $\mathrm{H}=3.0 \mathrm{~m}$.

Table 1: Comparison of bending moment

\begin{tabular}{|c|c|c|c|}
\hline & Stupisin L. & $\begin{array}{c}\text { Straughan } \\
\text { W. [5] }\end{array}$ & $\begin{array}{c}\text { SCAD } \\
\text { office }\end{array}$ \\
\hline $\begin{array}{c}\text { The value of } \\
\text { the bending } \\
\text { moment, N.m }\end{array}$ & 10,14 & 17,62 & 5,0 \\
\hline
\end{tabular}

The difference in the results of the second and the third column explains the differences in geometry of the structure (shallow shell with minimal rise and plate). Calculation of the finite element method in a program complex «SCAD - office» is executed (400 triangular finite elements). Finite - element numerical procedure based on an approximation of a triangular area of a fourth-order polynomial. Geometrically nonlinear solution of the problem is carried out the method of successive loadings.

\section{OPTIMIZATION METHOD}

Research shows that there are various combinations of the values of shell thickness and the parameter that characterizes the vertical deformation profile within the soil continuum at which the stress in shell is the same [06, 07, 08, 09, 10]. Similar relationships were found for the critical load. Therefore, it is possible to find the optimal combination of these parameters with limiting the value of the stress, critical load the thickness (volume) of the shell or the characteristics of the elastic foundation:

$\left\{\begin{array}{l}\sigma(\gamma, t) \rightarrow \sigma_{\min } ; \\ V(t)-V_{0} \leq 0 .\end{array}\right.$
$\left\{\begin{array}{l}V(t) \rightarrow V_{\min }, \\ \sigma(\gamma, t)-\sigma_{0} \leq 0 .\end{array}\right.$
$\left\{\begin{array}{l}p_{c r}(y, t) \rightarrow p_{\max } ; \\ V(t)-V_{0} \leq 0 .\end{array}\right.$
$\left\{\begin{array}{l}V(t) \rightarrow V_{\min }, \\ p_{c r}(y, t)-p_{0} \geq 0 .\end{array}\right.$

Where:

$V\left(x_{i}\right)$ - volume function,

$\sigma\left(x_{i}\right)$ - stress function,

$p\left(x_{i}\right)$ - critical load function.

This will make it possible to optimize geometrically nonlinear shallow shells on an elastic foundation due to optimal form distribution when there is a need to:

- optimize stress in the median surface of a shell for any kinds of support with a limitation on the volume of used material;

- optimize critical load of a shell for any kinds of support with a limitation on the volume of used material;

- optimize the volume of a used material for any kinds of support at preset values of the stresses or critical load.

This can ensure considerable savings in the weight of building structures. The dependences and patterns allow identifying areas for optimization of shallow shells on elastic foundation.

The graphs show, that the stress increases when the values of the parameter that characterizes the vertical deformation profile within the soil continuum grow up and the values of the thickness decrease. Obviously, the volume of the shells decreases with decreasing thickness, and 
does not depend on the parameter that characterizes the vertical deformation profile within the soil continuum.

Each of the parameters of shell can be used as criteria of optimization. Therefore, the task is very difficult. The correct formulation of the problem and the choice of optimization algorithm is possible to solve these problems.

The optimization algorithm is built on a combination of techniques gradient method and random search method. Penalty functions method of constrained function minimization is used.

After normalization parameters field of admissible values of the objective function:

$$
x_{i}=\frac{X_{i}}{x_{c i}} ; i=\overline{1, n} \text {, }
$$

where $x_{i}$ - a new space of dimensionless parameters,

$X_{i}$ - the former space of dimensionless parameters,

$x_{c i}$ - the characteristic sizes of which are selected from the condition,

$$
\frac{\partial V}{\partial x_{i}}=-|V|, i=\overline{1, n} \text {, }
$$

in the initial point of the search.

Steepest descent from the acceleration can be described by recurrent relations:

$$
\left\{\begin{array}{l}
\Delta x_{i}^{(1)}=h / n ; \\
\Delta x_{1}^{(k+1)}=\alpha \Delta x_{i}^{(k)},
\end{array}\right.
$$

while, $V^{(k)}(\vec{x}) \triangleleft V^{(k-1)}(\vec{x})$, and $\vec{x}_{i}^{(k)} \in \Omega ; i=\overline{1, n}$.

In the case of increasing or exit point for a boundary, random search algorithms are used. The minimum is sought on a set of points uniformly distributed inside a cube with sides $2 \mathrm{~h}$ and centered at the point with the smallest criterion value. In each of the next series of $t$ samples decreases the size of the cube:

$h^{(k)}=c h^{(k-1)}$,

were $0<\mathrm{c}<1$.

In each of the next series of random samples averaged steps and moved in the direction of minimum.

$$
\Delta x_{i}^{(k+1)}=\alpha\left[x_{i}^{(k)}-x_{i}^{(k-N)}\right] \frac{n}{N}, i=\overline{1, n} .
$$

After carrying out the specified number of samples, the search stops.

For solving problems (38) - (41) used a penalty function method. If the constraints in the problems are accurate to some small:

$$
y(\vec{x}) \leq \varepsilon_{k}, k=\overline{1, m},
$$

the quality criterion can be represented in the form:

$$
\bar{V}(\vec{x})=V(\vec{x})+\sum_{k=1}^{m} L_{k}\left(V_{k}\right) V(\vec{x}),
$$

where $L_{k}\left(V_{k}\right)$ - the penalty coefficients are selected as follows:

$L_{k}\left(V_{k}\right)=\left\{\begin{array}{l}L_{k} \text { if } V_{k}>0, \\ 0 \text { if } V_{k} \leq 0 .\end{array}\right.$

The positive coefficients must satisfy the relations:

$$
L_{k}\left(V_{k}\right) \geq \frac{V^{*}}{\varepsilon_{k}}, \quad k=\overline{1, m} \text {. }
$$

$\mathrm{V}^{*}$ - the accepted absolute value $\mathrm{V}(\mathrm{x})$ in the initial point of the search.

In some cases just due to a rational thickness distribution it is possible to save up to $25 \%$ of its material with a considerable weight reduction of the structure.

\section{CONCLUSION}

This methodology can be applied to determine stresses for geometrically nonlinear shallow shells on an elastic foundation of variable shape of the middle surface, thickness of shell and the characteristics of the elastic foundation. In some problems, the numerical method provides greater accuracy and adaptability compared with the finite element method. A rational thickness distribution it is possible to save up a significant of its material with a considerable weight reduction of the structure.

This work was supported by the grant of the President of the Russian Federation [MK9203.2016.8].

\section{REFERENCES}

1) Nie G, Chan C, Yao J, He X (2009): Asymptotic solution for nonlinear buckling of orthotropic shells on elastic foundation, AIAA Journal, 47- 7, pp. 1772-1783. 
2) Stupishin L, Kolesnikov A (2014): Geometric nonlinear shallow shells for variable thickness investigation, Advanced Materials $\mathrm{Re}$ search, 919-921, pp. 144-147.

3) Trushin S, Zhavoronok S (2002): Nonlinear analysis of multilayered composite shells using finite difference energy method, Space Structures 5 proceedings of the Fifth International Conference on Space Structures, held at the University of Surrey, pp. 1527-1533.

4) Stupishin L, Kolesnikov A (2014): Reconstruction of shallow shells for increase bearing capacities and operating characteristics, Applied Mechanics and Materials, 580-583, pp. 3062-3065.

5) Straughan W (1990), Analysis of plates on elastic foundations, Texas Tech University, p. 85.

6) Stupishin L, Kolesnikov A, Nikitin K (2017): Variable form forming investigation for flexible shallow shells on circular base, Asian journal of civil engineering (BHRC) vol.18,№.2 pp.163-171
7) Stupishin L.U., Nikitin K. (2014) Mixed finite element of geometrically nonlinear shallow shells of revolution. Applied Mechanics and Materials. T. 501-504. pp. 514-517.

8) Andreev V, Avershyev A (2013): Stationary problems of moisture-elasticity for inhomogeneous thick-walled shells, Advanced Materials Research, 671-674, pp. 571-575.

9) Stupishin L, Kolesnikov A, Ozerova T (2013): Investigation of Stressed-Strained State of Shallow Geometric Nonlinear Shells On The Round Plan of Variable Shape at Different Types Of Loading, Industrial and Civil Construction. № 5. pp. 33-34.

10) Nath $Y$, Jain $R$ (1983): Non-linear dynamic analysis of shallow spherical shells on elastic foundations, International Journal of Mechanical Sciences, 25,

Paper sent to revision: 19.07.2017.

Paper ready for publication: 13.09.2017. 